\title{
Magnetic Effects in the Thermodynamics of the Process of Thinking
}

\author{
Aibassov Yerkin Zhakenovich ${ }^{1 *}$, Savizky Ruben ${ }^{2}$, Yemelyanova Valentina ${ }^{1}$, Shakieva Tatyana ${ }^{1}$, Tussupbaev \\ Nessipbay ${ }^{1}$, Bulenbayev Maxat ${ }^{1}$ and Blagikh Evgeniy ${ }^{1}$ \\ 1. Research Institute of New Chemical Technologies and Materials, Kazakh National University Al-Farabi, Almaty 005012,
} Kazakhstan

2. Department of Chemistry, the Columbia University, NewYork 005012, USA

\begin{abstract}
The authors have shown that the process of information and thinking can be modeled on the basis of chemical thermodynamics. The authors offer general equations to calculate the thinking of the work of judgment the $\mathrm{L}$ and of entropy solutions $\mathrm{G}$ in the presence of a magnetic field. As a result, studies have shown that the magnetic effects strongly influence the thermodynamics of the process of thinking.
\end{abstract}

Key words: Magnetic effect, thermodynamics, process of thinking, Shonon equation.

\section{Introduction}

The human brain is the most mysterious organ that controls thought processes.

In recent years, many works devoted to the thermodynamics of the thinking process [1-6].

The authors have previously been shown that the thermodynamic functions of internal energy $d U$ and free energy $d F$ in the presence of a magnetic field [5]:

$$
\begin{gathered}
d U=T d S+1 / 4 \pi H d B \\
d F=-S d T+1 / 4 \pi H d B
\end{gathered}
$$

It was also shown that the Nernst equation in a magnetic field change, and is described by the Eq. (3) [6]:

$$
E=E^{0}+R T / n F \ln a_{O x} / a_{\text {Red }}+1 / 4 \pi H d B
$$

The purpose of this work is an attempt to study the magnetic effects in thermodynamics thought processes and to find patterns of thinking mechanism in living organisms.

\section{Theory}

Thinking - certainly a biological phenomenon, and

Corresponding author: Aibassov Yerkin Zhakenovich, professor, research fields: metalorganic chemistry of uranium and thorium, As, Sb and $\mathrm{Bi}$. should therefore be subject to the atomic and molecular description. Science has established that the complex DNA and RNA molecules are capable of performing the function of storage $\mathrm{n}$ transmission of information is even more faith in the fact that the complex information processes, including thinking, made atomic-molecular mechanisms.

The fundamental thermodynamic properties of Shannon-type thinking is that the solution to the problem of information is not a process of spontaneous and necessary, i.e. running with decreasing free energy, but, on the contrary, requires the expenditure of work. This expresses the basic property information, that it is not deducible from the known data as a deduction - otherwise it would not be at all necessary, as always could be obtained from these data-but gives the new independent information that do not have believed to be reliable.

The process of logical thinking is similar to spontaneous thermodynamic process: In both cases, the original particle system (information) to be converted to a finite system of new particles or inferences. As spontaneous process in the thermodynamics with decreasing capacity of the free 
energy, and always leads to a more stable state. Spontaneous logical process flows down the free energy and gives the thermodynamically stable as a result of the withdrawal or deduction. The process of thinking is described thermodynamically expression:

Work Information: $I_{\text {Inform }}<0, \Delta \varphi_{\text {Inform }}<0$,

The work of judgment (solution):

$$
L_{\text {Solution }}>0, \Delta \varphi_{\text {Solution }}>0 \text {, }
$$

where $\Delta \varphi$ - the fall of the free energy in the act of information or diskursii.

Between these extremes there is an intermediate area in which the magnitude of the fall of the free energy is large enough to process the judgment was spontaneous, but not high enough for it to be unique. This is an ambiguous area of probabilistic thinking.

In connection with the (Eq. (4)) increase in free energy of the information and hence its instability, it has to be stored in anti-entropy devices - in memory, in the records of a particular type, including a variety of codes—otherwise it will inevitably dissipate.

\section{Results and Discussion}

Energy - is the ability to do the job, but also the ability to create and modify any type of order in nature.

Dynamic energy $E=Z \lambda-\ni$ energy action in a certain space, energy vektorization energy; $\Psi$-energy of the order.

Dynamic energy is manifested in two forms: vector $\mathrm{E}_{\mathrm{V}}=\mathrm{E} \eta$, giving direction and displacement of an object in its area of action and Brownian $E_{B}=E$ (1 $\eta)$, extending the scope and status of the process.

Hence the total energy $U_{\text {Total }}=E_{V}+E_{B}+\Psi$

The information in contrast to the thinking can not appear as a product of pure inferences from other data. It is impossible, without spending any work, just standing on the platform, by "pure" reasoning to know address of your friend.

Information data are logically independent of each other, it is as if from nowhere output set of "primary", independent data (in physical chemistry - the system of non-interacting particles).

Thinking (especially in its marginal, formal logical form) operates on the information data according to the laws of logic, and it resembles a chemical reaction "dissimilar" of the particles of the gas mixture to react with strictly defined laws.

The result of thinking is the conclusion that can be recorded. They include various elements of the original information.

Thinking there where begins the act of judgment as a result of a conscious selection of the source data come parcels in the form of some data (information), self-evident position (axioms) and certain assumptions (hypotheses), and subjecting them to an algorithm constructed in accordance with the laws of logic.

With this information system, axioms and hypotheses thinking process always leads to a definite conclusion. This is a very important property of thinking. It is similar to some spontaneous process, the outcome of which, regardless of the physical and chemical properties of the medium, where it occurs, is always the same!

It's like a roller coaster movement of rail road. Point of arrival is not dependent on anything. It is defined only by the desire to move the system to a stable state.

Thus, the thinking process is modeled on the basis of chemical thermodynamics in the form of spontaneous transition (Z-1) varieties of "chances" that are concentrated in a single cell in a k-th some sort of a drop in the free energy and entropy, expressed by the equation of thinking:

The work of judgment (Solution):

$$
L=\Sigma p_{i} \log p_{i}+\Delta \varphi^{o}=I_{\text {Vin }}+\Delta \varphi^{o}>0
$$

Entropy judgments (Solutions):

$$
G=\Sigma p_{i} \log p_{i}+\Delta H^{o}=H_{\text {Sannon }}+\Delta H^{o}>0
$$

The authors offer general equations to calculate the thinking of the work of judgment $L$ and entropy solution $G$ in the presence of a magnetic field:

$$
\begin{gathered}
L=I_{V i n}+\Delta \varphi+1 / 4 \pi H d B, \\
G=H_{\text {Sannon }}+\Delta H+1 / 4 \pi H d B,
\end{gathered}
$$

The final result of the act of thinking - a conclusion 
or inference - and there is a stable state of mind in the thermodynamic sense of the word.

Amazing uniqueness of the final result of mental activity proves it strictly directed, "spontaneous", the vector character, apparently independent from the chaos of Brownian motion of atoms and molecules that make up the substance of the brain or any other mechanism where there is thinking.

Inference can be repeated numerous times with the same result. For example, you can repeat as often as proof of the Pythagorean theorem. This means that the probability of a thermodynamic system, which carries out the process of thinking is always equal to one, which corresponds to the only possible way of thinking of those responsible for the microparticles, their full order and they are not susceptible to thermal chaos.

\section{Conclusion}

The analysis shows that the thinking process is modeled on the basis of chemical thermodynamics in the form of spontaneous transition (Z-1) varieties of "chances" that are concentrated in a single cell in a k-th some sort of a drop in the free energy and entropy, expressed by the equation of thought.

The authors offer general equations to calculate the thinking of the work of judgment $L$ and entropy solution $G$ in the presence of a magnetic field:

$$
\begin{gathered}
L=I_{V i n}+\Delta \varphi+1 / 4 \pi H d B, \\
G=H_{\text {Sannon }}+\Delta H+1 / 4 \pi H d B,
\end{gathered}
$$

The authors have shown that the process of thinking can be modeled on the basis of chemical thermodynamics. The authors offer general equations to calculate the thinking of the work of judgment the Land of entropy solutions $G$ in the presence of a magnetic field. As a result, studies have shown that the magnetic effects strongly influence the thermodynamics of the process of thinking.

The consequence of the above is the ability to save the information entropy and speed of thinking in three dimensions, so the speed of thought to increase by several orders of magnitude.

Research in this direction will be continued.

\section{Acknowledgments}

The authors would like to thank Lynn C. Francesconi (Hunter College CUNY), Peter C. Burns (Notre Dame University, Indiana) and Chistopher L. Cahill (George Washington University) for discussion of the results.

\section{References}

[1] Claude, E. 1948. "Shannon: A Mathematical Theory of Communication." Bell System Technical Journal 27: 379-423.

[2] Claude, E. S., and Warren, W. 1949. The Mathematical Theory of Communication. The University of Illinois Press, Urbana, Illinois.

[3] Claude, E. S. 1950. Programming a Computer for Playing Chess, Philosophical Magazine, Ser. 7, 41: 314.

[4] David, L. 1983. Computer Gamesmanship: Elements of Intelligent Game Design. Simon \& Schuster.

[5] Aibassov, Y., and Yemelyanova, V. 2014. "Study of Changes in the Thermodynamic Functions in a Magnetic Field." J. Chem. Chem. Eng. 8: 119-22.

[6] Aibassov, Y., and Yemelyanova, V. 2015. "Derivation of the Equation Nernst-Aibassov in a Magnetic Field." $J$. Chem. Chem. Eng. 9: 218-220. 